\title{
Smad9 is a key player of follicular selection in goose via keeping the balance of LHR transcription
}

Daolun $\mathrm{Yu}^{1,4, *}$, Fanghui Chen ${ }^{2, *}$, Li Zhang ${ }^{1}$, Hejian Wang ${ }^{1}$, Jie Chen ${ }^{1}$, Zongmeng Zhang ${ }^{1}, \mathrm{Jie}^{2}{ }^{1}$, Chaofeng Xing ${ }^{1}$, Honglin $\mathrm{Li}^{3}$, Jun $\mathrm{Li}^{1, \square}$, Yafei Cai ${ }^{2, \square}$

${ }^{1}$ College of Life Sciences, Anhui Provincial Key Lab of the Conservation and Exploitation of Biological Resources, Anhui Normal University, Wuhu 241000, China

${ }^{2}$ College of Animal Science and Technology, Nanjing Agricultural University, Nanjing 210095, China

${ }^{3}$ Department of Biochemistry and Molecular Biology, Augusta University, Augusta, GA 30912, USA

${ }^{4}$ College of Biotechnology and Pharmaceutical Engineering, West Anhui University, Lu'an 237012, China

"These authors contributed equally to this work

${ }^{\square}$ Authors for corresponding: Yafei Cai, Tel: +86-13151568780, E-mail: ycai@njau.edu.cn Jun Li, Tel: +86-18855356196, E-mail: lijunplant@163.com

\section{SUMMARY STATEMENT}

To study the hierarchical development mechanism of avian follicle, new strategies can be found to improve the egg production of low-yielding poultry, such as geese.

\section{ABSTRACT}

The egg production of poultry depends on follicular development and selection. However, the mechanism of selecting the priority of hierarchical follicles is completely unknown. Smad9 is one of the important transcription factors in BMP/Smads pathway and involved in goose follicular initiation. To explore its potential role in goose follicle hierarchy determination, we first blocked Smad9 expression using BMP typelreceptor inhibitor LDN-193189 both in vivo and in vitro. Unexpectedly, LDN-193189 administration could dramatically suppress Smad9 level and elevate egg production (7.08 eggs / bird, $P<0.05)$ of animals, and the estradiol $\left(\mathrm{E}_{2}\right)$ and luteinizing hormone receptor (LHR) level were significantly increased $(P<$ 0.05), but the progesterone $\left(\mathrm{P}_{4}\right)$ and follicle stimulating hormone receptor (FSHR) 
mRNA remain unchanged. Surprisingly, Smad9 knockdown notably attenuated $(P<$ 0.05) in $\mathrm{E}_{2}, \mathrm{P}_{4}$, FSHR and LHR level in goose granulosa cells (gGCs). Further chromatin immunoprecipitation (ChIP) assay of gGCs revealed that Smad9, served as a sensor of balance, bound to the LHR promoter regulating its transcription. These findings demonstrated that Smad9 is differentially expressed in goose follicles, and acts as a key player in controlling goose follicular selection.

KEY WORDS: Smad9 gene, granulosa cells (GCs), follicular hierarchy development, ovulation, goose

\section{INTRODUCTION}

The geese (Anser cygnoides) are cultivated widely in China as one of most important farm poultries. However, the poor fertility seriously restricts its further application. The avian ovary contains large number of follicles during the laying period, which can be divided into two types: prehierarchical follicles and preovulatory follicles. The prehierarchical follicles comprise three types of follicles: small white follicles (SWF, 1-2 mm), large white follicles (LWF, 3-5 mm) and small yellow follicles (SYF, 6-9 $\mathrm{mm}$ ). The preovulatory follicles consist of yolk-filled follicles at five stages (F5, F4, F3, F2, F1, from small size to large size) in laying hens (Etches and Petitte, 1990; Jia et al., 2010). Folliculogenesis involves a series of events during which a growing follicle either develops to the ovulation stage or undergoes atresia (McGee and Hsueh, 2000). The maturation and ovulation of the follicle is strictly regulated by a variety of endogenous factors.

Bone morphogenetic proteins (BMPs) belong to the transforming growth factor $\beta$ (TGF- $\beta$ ) superfamily and elicit their effects through activation of type-1 and type-2 serine/threonine kinase receptors on the target cells (Su et al., 2009). BMP typel receptor kinases phosphorylate various downstream receptor-regulated Smads (R-Smads) upon ligand binding (Kawabata et al., 1998; Schmierer and Hill, 2007). R-Smads include Smad1, Smad5 and Smad9 (also known as Smad8) can be 
phosphorylated by BMP type I receptor kinase form complexes with Smad4, a Co-Smad, and bind to the responsive element (BRE) to regulate the transcription of target genes (Massague et al., 2005). Therefore, R-Smads are vital for intracellular transmission of BMP signals.

BMP/Smads signaling pathways were reported to play important regulatory roles in the development, atresia and selection of the follicles in mammals (Costello et al., 2009; Haugen and Johnson, 2010; Knight and Glister, 2003; Miyoshi et al., 2007; Nilsson and Skinner, 2003; Pangas and Matzuk, 2004; Wang et al., 2010; Ying et al., 2001). However, there are clear differences in the follicular development between birds and mammals, and it is unclear whether the BMP/Smads system still plays a role in the folliculogenesis of birds. Smad9 is one important member of the Smads family proteins, although their function is largely unknown. Previous studies have shown that activation of BMP signaling pathway could increase Smad9 expression (Tsukamoto et al., 2014). Interestingly, Smad9 contains a special linker region that can result in its markedly lower transcriptional activity than Smad1 and Smad5. In turn, Smad9 overexpression can reduce the activity of BMP in a dominant negative manner through inhibiting the transcription of target genes (Tsukamoto et al., 2014). Therefore, Smad9 is crucial for the balance of BMP/Smads signaling. In addition, Smad9 was found to be involved in the follicular initiation but was silent during maturation process in goose (Xu et al., 2015). However, whether it is involved in regulating follicular development and the underlying mechanism is still unclear. To address these issues, Chinese Wanxi White Goose, which have a low annual egg yield of only about 20-25 eggs, were chosen in this study. The distribution of Smad9 in the ovary was investigated and then its expression was manipulated by using LDN-193189, a kinase inhibitor of BMP typelreceptors. Finally, we analyzed the hormone secretion, receptor-expression and the number of ovulation. Our results showed that Smad9 can regulate $\mathrm{E}_{2}$ secretion and LHR transcription both in vivo and in vitro. Most importantly, we found that attenuation of Smad9 expression during laying stage could significantly elevate goose egg production. This study shed new light on the role of 
Smad9 in avian follicular development and ovulation.

\section{RESULTS}

\section{Smad9 expression in the GCs layer of follicles}

To analyze the expression of Smad9 in goose ovary, the ovaries were collected from Chinese Wanxi White Goose in laying period. We can see a number of prehierarchical follicles and only one preovulatory follicle at different development stages in the mature goose ovary (Fig. 1A). Hematoxylin and eosin (H\&E) staining was further carried out to analyze the histological structure of follicular wall. The results revealed that the thickness of GCs layer showed a trend of first increased and then decreased, which reached the maximum at F3. No significant difference in the thickness of GCs layer among SWF, F5 and F2 as well as difference between SYF and F1 was detected, however significant differences were shown among F3, F4, F1 and SWF $(P<0.05)$ (Fig. 1B). Finally, immunohistochemical analysis showed that Smad9 was specifically expressed in the GCs layer of follicles, and no signaling was detected in other parts of the ovary (Fig. 1C). Based on these observations, it can be concluded that the morphological of GCs layer showed regular changes with follicles development and the Smad9 was expressed only in GCs layer in goose follicles.

\section{Smad9 activation can be transferred to the nucleus in gGCs}

Smad9 is a transcription factor which must be present in the nucleus and play its biological functions. In order to locate total Smad9 and phosphorylated Smad9 (p-Smad9, the active form of Smad9 protein) expression in gGCs, immunofluorescence techniques are used to determine their distribution. The results showed that Smad9 protein is only present in the cytoplasm and p-Smad9 protein is present in the cytoplasm and nucleus (Fig. 2A,B). These results also mean that Smad9 can be translocated to the nucleus to play its biological role after activation.

\section{Differential expression of Smad9 in hierarchical follicles}

To test whether the expression of Smad9 changes with the process of follicle 
development, we further analyzed its expression in follicles at different stages by quantitative real-time polymerase chain reaction (qRT-PCR) method. The results showed that in control group, Smad9 expression is gradually decreased in the prehierarchical follicles, which tended to decrease in the preovulatory follicles but with a fluctuation in the F4. It expression touched the bottom in the F1 follicle (Fig. 3A). In contrast, Smad9 expression is inhibited in LDN-193189 treated group, especially in the preovulatory phase with an exception of SYF (Fig. 3A,B). Moreover, the differences of Smad9 expression among SWF, LWF, SYF, F4 and F1 were significant while they were very significant among F5, F3 and F2. In addition, Smad9 transcription and translation levels were consistent in respective group, the same as to total Smad9 and p-Smad9 (Fig. 3C). In a word, these results revealed that Smad9 is differentially expressed along with the process of follicle development.

\section{Blockage of Smad9 promotes the LHR expression and $E_{2}$ biosynthesis as well as egg production in vivo}

FSHR and LHR were reported to be involved in follicular development and ovulation (Camp et al., 1991; Ji et al., 2014). To investigate whether LDN-193189 treatment has an effect on the expression of these two hormone receptors, we first analyzed their expression in hierarchical follicles from laying geese by qRT-PCR method (Fig. 4A,B). The results showed that FSHR expression were different with the process of follicle development, which was not clearly affected by LDN-193189 treatment (Fig. 4A). The results also showed that LHR expression gradually increases in prehierarchical follicles until SYF, in which it was significantly higher than in other follicles $(P<0.05)$, then decreased in F5 but increased in F4, followed by a gradual decline from F3 to F1. In contrast, in LDN-193189 treatment groups, the LHR expression in prehierarchy follicles was similar in control group, however, it was different in preovulatory follicles because it gradually increased. There is no significant difference between SYF and F1, but it was significantly higher than that in any other follicles. In both the control and LDN-193189 treatment groups, the differences were not significant in SWF and LWF, and significant among F5, F3 and 
F2 $(P<0.05)$, and very significant among SYF, F4 and F1 $(P<0.01)$ (Fig. 4B).

$\mathrm{E}_{2}$ and $\mathrm{P}_{4}$ were two representative steroid hormones that regulate follicular development and ovulation. To study whether the treatment of LDN-193189 has any effects on the expression of these two steroid hormones, the $\mathrm{E}_{2}$ and $\mathrm{P}_{4}$ in the serum samples from laying period geese were measured by ELISA. The results showed that $\mathrm{E}_{2}$ levels in LDN-193189 treatment group were significantly higher than that in control group $(P<0.05)$ (Fig. $4 \mathrm{C})$. However, $\mathrm{P}_{4}$ levels were significantly higher in the treatment group than that in the control group only in 14 days post LDN-193189 treatment $(P<0.05)$. Although there were no significant differences were found at other points of time, a slight increase was seen after treatment with LDN-193189 (Fig. 4D). Most importantly, the average egg production of LDN-193189 treatment group (31.86 eggs) was significantly higher than that of control group $(24.78$ eggs $)(P<0.05)$ (Fig. 4E). In conclusion, blockage of Smad9 in vivo significantly affects $\mathrm{E}_{2}$ secretion in serum and folliclar LHR gene expression which is closely related to follicular development and ovulation, eventually contributing to promoting egg production.

\section{Blockage of Smad9 promotes the LHR expression and $E_{2}$ biosynthesis and cell proliferation of GCs cultured in vitro}

GCs play a vital role in the development and maturation of follicles (Tilly et al., 1991).

To further elucidate the effects of Smad9 on the development of GCs, we examined the relationship between Smad9 expression and hormone synthesis, hormone receptor expression and cell proliferation after primary cultured gGCs being treated with BMP-4, BMP-4 / LDN-193189, Smad9-siRNA for 48 h respectively (Fig. 5). Smad9 mRNA and protein were detected by qRT-PCR and western blotting, respectively. The results showed that Smad9 gene was knocked down by Smad9-siRNA (Fig. S1). Smad9 expression was significantly higher in BMP-4 group than that in blank $(P<$ 0.05), and that in Smad9-siRNA group and BMP-4 / LDN-193189 group were significantly lower than that in blank $(P<0.05)$. The expression of $\mathrm{p}$-Smad9 was similar to the expression of total Smad9 in those groups (Fig. 5A,B).

The qRT-PCR analysis showed that FSHR expression were no significant 
difference in BMP-4 group and BMP-4 / LDN-193189 group compared with blank, however, there was significantly lower in Smad9-siRNA group than in blank $(P<$ 0.05). LHR expression in BMP-4 / LDN-193189 was significantly higher than in the blank $(P<0.05)$, and there was no significant difference in BMP-4 compared with blank. However, it was significantly lower in Smad9-siRNA than that in blank $(P<$ 0.05) (Fig. 5C).

$\mathrm{E}_{2}$ and $\mathrm{P}_{4}$ in the supernatants were analyzed by ELISA showed that $\mathrm{E}_{2}$ levels were significantly higher in BMP-4 / LDN-193189 than that in the blank $(P<0.05)$, no significant difference in BMP-4 group compared with the blank. $\mathrm{P}_{4}$ levels in BMP-4 group and BMP-4 / LDN-193189 group were no significant difference in comparison with the blank. However, the concentration of $\mathrm{E}_{2}$ and $\mathrm{P}_{4}$ were significantly lower in Smad9-siRNA than in the blank $(P<0.05)$ (Fig. 5D, E).

The growth of GCs was examined by CCK-8 assay. The results showed that BMP-4 / LDN-193189 markedly prompted the growth of GCs compared with the blank $(P<0.05)$. And we also found that Smad9-siRNA markedly inhibited the growth of GCs compared with the blank $(P<0.05)$. In addition, there was a slight increase in promoting cell proliferation efficiency in BMP-4 group compared with blank, but no significant difference (Fig. 5F). In conclusion, Smad9 plays an important role in the development of GCs by affecting the secretion of $E_{2}$ and LHR expression, which can promote cell proliferation.

\section{Smad9 regulates LHR transcription in gGCs}

Unexpectedly, FSHR in vivo and in vitro experiments showed no significant difference, which promoted us to test whether Smad9 binds to LHR promoter by ChIP assay. Our results showed that Smad9 can bind to the promoter region of LHR and regulating its transcription (Fig. 6A). It was remarkable that Smad9 expression increased post-stimulating with BMP-4 in gGCs and also increased its combined efficiency with LHR promoter (Fig. 6B). Intriguingly, the efficiency of Smad9 bonding to the LHR promoter was not reduced because Smad9 phosphorylation was inhibited, meanwhile, LHR transcription increase was observed after inhibiting 
Smad9 protein phosphorylation with LDN-193189 (Fig. 6B,C). In conclusion, this inner molecular mechanism shows us that Smad9 can directly regulate LHR transcription in gGCs. In addition, the proposed pathway of Smad9-mediated LHR transcription is summarized in Figure 7.

\section{DISCUSSION}

To our best knowledge, the present study is the first to characterize the expression and localization of Smad9 in goose hierarchical follicles and follicular GCs during the laying stage. Our results clearly showed that Smad9 is majorly located in the cytoplasm while the p-Smad9 is translocated to the nucleus as was described in the previous study (Massague, 2012). Also, for the first time our results revealed that appropriate level of Smad9 expression in gGCs during reproductive cycle is positively correlated with $\mathrm{E}_{2}$ secretion, LHR transcription and cell proliferation. In addition, our $H \& E$ staining results indicated that the thickness of the GCs layer changed with follicular development, which further showed the importance of GCs to follicular development.

\section{Particularity of Smad9 distribution}

GCs play an essential role in the development, maturation and ovulation of follicle (Fitzharris and Baltz, 2006; Makita and Miyano, 2014; Orisaka et al., 2009; Zhang et al., 2011). It has previously been indicated that interrupted endogenous BMP/Smads signaling influence the growth and steroidogenesis of porcine GCs (Wang et al., 2010). Smad9 is an important downstream transcription factors of BMP/Smads signaling pathway which was ubiquitously expressed in different tissues and mediates various physiological processes (Massague and Wotton, 2000). In our study, the changing localization pattern of Smad9 in developing follicles GCs indicated the implication of BMP/Smads signaling in goose ovary. Accordingly, we may infer that similar to other mammals, BMP/Smads system in regulating female reproduction also occur in geese. We can also infer that the presence of Smad9 in GCs to facilitate the reception and transduction of signals inside and outside of the follicle. 


\section{Smad9 difference expression in the hierarchical follicles of goose}

In this study, hierarchy follicles with continuous size have been successfully obtained from laying goose. Further studies demonstrated that the morphology and quantity of the follicles used in our study were similar to that from other birds (Etches and Petitte, 1990). We separated the follicular GCs and detected the expression of Smad9. For the first time the spatiotemporal patterns of Smad9 expression in the hierarchical follicles of goose were revealed. Our data showed that Smad9 is differentially expressed in different hierarchical follicles at both transcription and translation levels. Also, our present results further extended our previous findings on the expression of Smad9 in goose ovary (Xu et al., 2015). The differential expression of Smad9 during follicular hierarchy establishment indicates its involvement in establishing or maintaining the follicular hierarchy and regulating follicle development.

\section{Smad9 and follicular development and ovulation}

It is well known that the more the follicles that been chosen to enter the hierarchy, the longer the egg laying period and higher production of poultry. The development of avian follicles is a complex process, which is regulated by different hormones and signaling from various receptors such as $\mathrm{E}_{2}$ and $\mathrm{P}_{4}$, FSHR and LHR (Caicedo Rivas et al., 2016; Calvo and Bahr, 1983; Ji et al., 2014; Jia et al., 2010; McGee and Hsueh, 2000; Qin et al., 2013; Regan et al., 2015; Wei et al., 2013). In this study, the BMP typelkinase inhibitor, LDN-193189, was used to regulate Smad9 expression in vivo (Cannon et al., 2010; Derwall et al., 2012; Lee et al., 2011; Mayeur et al., 2015; Yu et al., 2008). We found that post treatment of LDN-193189, Smad9 expression was downregulated in hierarchical follicles, especially in the preovulatory phase. Meanwhile, we also found that the $\mathrm{E}_{2}$ and LHR levels were significantly increased, the $\mathrm{P}_{4}$ and FSHR levels were not changed. The results from our in vitro experiment further verified the reliability of results from in vivo. Our data also indicated that LHR expression in LDN-193189 treatment group from in vivo was consistent with high 
yield poultry chickens (Liu and Zhang, 2008). Intriguingly, we were surprised to find that the average egg production was significantly increased in LDN-193189 treatment geese compared with that in the control group $(P<0.05)$. In addition, we also found the efficiency of cells proliferation in BMP-4 / LDN-193189 group from in vitro was significantly higher than that in the blank group $(P<0.05)$. Both in vivo and in vitro results indicated that the expression of regulating Smad9 can directly affect $\mathrm{E}_{2}$ secretion, LHR transcription and cell proliferation, which finally contributes to the increased egg production. Unexpectedly, the expression of FSHR, LHR, $\mathrm{E}_{2}$ and $\mathrm{P}_{4}$ as well as GCs growth were all significantly decreased after Smad9 knock down. Our data also suggested that Smad9 inhibition may indirectly affect the other unknown mechanisms, which changes the expression of FSHR and LHR, $\mathrm{E}_{2}$ and $\mathrm{P}_{4}$ secretion and cell proliferation, remain to be further studied. Thus, our data strongly suggests that inhibition of Smad9 is a promising strategy with great potential for elevating goose egg production in the future.

\section{Smad9 and LHR transcription}

The transition from prehierarchical follicles to preovulatory follicles is accompanied by a shift from FSHR dominance to LHR dominance in follicular development (Johnson and Woods, 2009). Therefore, LHR is critical to the ovulation of mature follicle. Our ChIP assay results showed that Smad9 can directly bind to the genomic promoter region of LHR and regulate its transcription. Although Smad9 binding efficiency was higher in BMP-4 group than that in BMP-4 / LDN-193189 treatment group, the expression of LHR was lower in BMP-4 group at mRNA level. Serving as a sensor of LHR transcription, Smad9 can keep the balance of LHR transcription to some extent, and its high or low level doesn't work in follicular maturation. A recent report also showed that when Smad9 is expressed at high level, it could suppress BMP activity without inhibiting the phosphorylation of R-Smad (Tsukamoto et al., 2014). Taken together, these data indicate that Smad9 is the key regulator of LHR transcription in laying goose. 


\section{Conclusions}

The present study demonstrates that: (1) Smad9 is expressed in GCs of goose follicles; (2) Smad9 is differentially expressed in goose hierarchical follicles; (3) Smad9 regulates $E_{2}$ secretion and cell proliferation; (4) Smad9 can bind to the promoter region of LHR and regulate its transcription, which is served as a sensor of balance.

\section{MATERIALS AND METHODS}

\section{Animals, tissue harvest and serum samples collection}

Twenty-four laying period female Chinese Wanxi White Geese (from Anhui Sanyuan Breeding Co., Ltd.) were used in this study. The geese were divided into two groups and were treated with LDN-193189 (0.15 mg-1 kg/day) via wing vein $(\mathrm{n}=12)$ or with control saline $(\mathrm{n}=12)$ once a day for 10 successive days. Ten sequential blood samples were obtained at weekly interval a week after treatment. The blood samples were centrifuged at $1,500 \mathrm{rpm}$ for $20 \mathrm{~min}$ at $4^{\circ} \mathrm{C}$ and the serum samples were restored at $-80^{\circ} \mathrm{C}$ until use. All animals had free access to water and feed. The egg production was recorded during the geese laying period. To obtain follicles from the mature ovaries, geese were sacrificed after ovipositionin the middle of a laying stage. Hierarchical follicles were collected and stored in PBS or liquid nitrogen for further experiments. The RNA and protein were isolated from the follicles after removing the yolk. All animal experiments were approved by the Institutional Animal Care and Use Ethics Committee of Anhui Normal University and performed in accordance with the "Guidelines for Experimental Animals" of the Ministry of Science and Technology (Beijing, China).

\section{Histological analysis, immunohistochemistry and immunofluorescence}

For histological analysis, briefly, hierarchical follicles were fixed in bouin's solution for $48 \mathrm{~h}$ and embedded with paraffin. Sections were sliced at $5 \mu \mathrm{m}$ thickness and each section was stained with H\&E (Boster, China) as described previously (Lam, 1997).

For immunohistochemistry, follicles were embedded in paraffin following standard 
protocols. The transverse sections $(5 \mu \mathrm{m})$ were incubated with antibody against Smad9 (1:1000; Abcam, USA) for overnight at $4^{\circ} \mathrm{C}$. The sections were then incubated with horseradish peroxidase-conjugated secondary antibodies followed by diaminobenzidine and counterstained with hematoxylin.

For immunofluorescence, GCs were grown in glass chamber slides. After treatments, cells were washed with PBS three times and fixed in $4 \%$ paraformaldehyde. For immunostaining, cells were incubated with the antibodies against Smad9 (1:1000; Abcam, USA) and p-Smad9 (1:800; CST, USA) for overnight at $4^{\circ} \mathrm{C}$. Slides were washed three times with cold PBS and incubated with donkey anti-rabbit IgG-FITC (1:1000; Beyotime, China) for $1 \mathrm{~h}$ at RT. Then, the slides were washed three times with cold PBS and cells were counterstained with DAPI (Beyotime, China). The immunofluorescent signals were examined with Olympus BX61 fluorescence microscope. All images were captured using the same settings and saved in the same format.

\section{GCs culture, design and transfection of Smad9-siRNA and supernatant Samples collection}

The GCs of SYF follicle were separated using the improved method as described by Gilbert et al (Gilbert et al., 1977). Then the cells were incubated at $39^{\circ} \mathrm{C}$ in a water-saturated atmosphere of 95\% air and 5\% CO2.

Six oligonucleotides were designed and synthesized by Shanghai GenePharma Co., Ltd according to the cDNA sequence of goose Smad9 (Table S1). The specificity of these sequences was then confirmed by transfection preliminary experiment according to the manufacturer's instructions (GenePharma, China). Finally, the siRNA sequence of Smad9 was identified as siRNA-1.

The cultured GCs were randomly divided into five groups: blank, BMP-4 50 ng/ml, BMP-4 50 ng/ml / LDN-193189 100 nM, Smad9-siRNA 16.7 nM and NC-siRNA $16.7 \mathrm{nM}$. Cells and culture supernatants were collected separately after treatment with serum-free medium for $48 \mathrm{~h}$. The cells were collected RNA and protein preparation, 
and supernatant were restored at $-80^{\circ} \mathrm{C}$ until use. Three replicates for each group and three independent repeated for each experiment.

\section{Cell proliferation assay}

A total of $3 \times 10^{3}$ GCs per well were incubated for $24 \mathrm{~h}$ in a 96-well plate. Cell proliferation was determined by using cell counting kit-8 (CCK-8) assay kit (Beyotime, China) after treatment with BMP-4, BMP-4 / LDN-193189, Smad9-siRNA and blank for $6 \mathrm{~h}, 12 \mathrm{~h}, 18 \mathrm{~h}, 24 \mathrm{~h}, 36 \mathrm{~h}$ and $48 \mathrm{~h}$ following the instruction from the manufacturer. The experiment was repeated for three times.

\section{Quantitative real-time PCR}

Total RNA was extracted from follicles or cultured GCs using Trizol (Invitrogen, USA) according to the manufacturer's instructions. The concentration and purity of isolated total RNA were determined using a standard spectrophotometer (K.O., China). The cDNA was obtained using reverse transcription kit (Tiangen, China) according to the manufacturer's instructions. The qRT-PCR was performed in a Bio-Rad CFX Manager System (BIO-RAD, USA) using a qRT-PCR kit and SYBR Green as the detection dye (Tiangen, China) according to the manufacturer's instructions. Primers used for qRT-PCR are listed in Table S2. Each sample was repeated three times, and the relative concentrations of all interested genes were calculated using the $2^{-\Delta \Delta}{ }^{\mathrm{Ct}}$ method (Livak and Schmittgen, 2001).

\section{Western blotting}

Total proteins were extracted from goose follicle GCs or cultured GCs with RIPA lysis buffer (Beyotime, China) and concentration was quantified using bicinchoninic acid (BCA) protein assay kit (Huamei, China) according to the manufacturer's instructions. Protein extracts were subjected to western blot analysis with rabbit anti-p-Smad9 antibody (1:1000; CST, USA), mouse anti-Smad9 antibody (1:1600; Abcam, USA), mouse anti-Gapdh antibody (1:500; Boshide, China) for primary 
antibodies, and anti-rabbit and anti-mouse IgG-HRP for secondary antibody, respectively.

\section{ELISA}

The levels of $E_{2}$ and $P_{4}$ in serum and supernatant samples were measured by goose $E_{2}$ and $\mathrm{P}_{4}$ ELISA kit (Halin, China) according to the manufacturer's instructions, respectively.

\section{ChIP assay}

Three dishes of GCs were used, one dish as blank, another two dishes were induced with BMP-4, one of which was stimulated by LDN-193189 after BMP-4 inducing for $12 \mathrm{~h}$, and then cultured for $48 \mathrm{~h}$. ChIP was conducted using a ChIP assay kit (CST, USA) following manufacturer's instructions. GCs were cross-linked with $1 \%$ formaldehyde (Sangon, China). Chromatin was digested with micrococcal nuclease (CST, USA). The immune complexes of DNA-protein were immune precipitated by p-Smad9 antibody (CST, USA). Normal rabbit IgG (CST, USA) was used as control. The purified DNA used as a template to expand the promoter region of the LHR by qRT-PCR. Six pairs of primers were designed in -1/-2000 bp of the LHR promoter region and listed in Table $\mathrm{S} 3$.

\section{Statistical analysis}

Two-tailed Student's $t$-test for two samples was used to calculate the $p$-values. The results were presented as mean \pm s.e.m, and asterisks or different letters in figures represents the level of significance as follows: “*” or "a, b, c.." $P<0.05$ were considered statistically significant, $* * P<0.01$ were considered statistically very significant.

\section{Acknowledgements}

We thank Anhui Sanyuan Breeding Co., Ltd. for providing the experimental geese. We also thank Dr Yongguang Yang for critical reading of the manuscript. 


\section{Competing interests}

The authors declare no competing or financial interests.

\section{Author contributions}

Y.F.C., D.L.Y. and Jun L. designed the experiments. D.L.Y., L.Z., H.J.W., Z.M.Z., Jie L., C.F.X. and F.H.C. performed the experiments. D.L.Y., J.C. and H.L.L. analyzed the data. D.L.Y. and Y.F.C wrote the manuscript.

\section{Funding}

This work was supported by Natural Science Foundation of China (31572265 and 81570094), Innovation Team of Scientific Research Platform in Anhui Province, A start-up grant from Nanjing Agricultural University (804090) and "Sanxin” Research Program of Jiangsu Province (SXGC[2016]312).

\section{Data availability}

Goose Smad9, FSHR, LHR and Gapdh gene data are available in NCBI with accession number XM_013192591, XM_013192472, XM_013192443 and XM_013199522, respectively.

\section{References}

Caicedo Rivas, R. E., Nieto, M. P. and Kamiyoshi, M. (2016). Effects of Steroid Hormone in Avian Follicles. Asian-Australasian journal of animal sciences 29, 487-499.

Calvo, F. O. and Bahr, J. M. (1983). Adenylyl cyclase system of the small preovulatory follicles of the domestic hen: responsiveness to follicle-stimulating hormone and luteinizing hormone. Biology of reproduction 29, 542-547.

Camp, T. A., Rahal, J. O. and Mayo, K. E. (1991). Cellular localization and hormonal regulation of follicle-stimulating hormone and luteinizing hormone receptor messenger RNAs in the rat ovary. Molecular endocrinology (Baltimore, Md.) 5, 1405-1417.

Cannon, J. E., Upton, P. D., Smith, J. C. and Morrell, N. W. (2010). Intersegmental vessel formation in zebrafish: requirement for VEGF but not BMP signalling revealed by selective and non-selective BMP antagonists. British journal of pharmacology 161, 140-149.

Costello, I., Biondi, C. A., Taylor, J. M., Bikoff, E. K. and Robertson, E. J. (2009). Smad4-dependent pathways control basement membrane deposition and endodermal cell migration at early 
stages of mouse development. BMC developmental biology 9, 54 .

Derwall, M., Malhotra, R., Lai, C. S., Beppu, Y., Aikawa, E., Seehra, J. S., Zapol, W. M., Bloch, K. D. and Yu, P. B. (2012). Inhibition of bone morphogenetic protein signaling reduces vascular calcification and atherosclerosis. Arteriosclerosis, thrombosis, and vascular biology 32, 613-622.

Etches, R. J. and Petitte, J. N. (1990). Reptilian and avian follicular hierarchies: models for the study of ovarian development. The Journal of experimental zoology. Supplement : published under auspices of the American Society of Zoologists and the Division of Comparative Physiology and Biochemistry 4, 112-122.

Fitzharris, G. and Baltz, J. M. (2006). Granulosa cells regulate intracellular pH of the murine growing oocyte via gap junctions: development of independent homeostasis during oocyte growth. Development (Cambridge, England) 133, 591-599.

Gilbert, A. B., Evans, A. J., Perry, M. M. and Davidson, M. H. (1977). A method for separating the granulosa cells, the basal lamina and the theca of the preovulatory ovarian follicle of the domestic fowl (Gallus domesticus). Journal of reproduction and fertility 50, 179-181.

Haugen, M. J. and Johnson, A. L. (2010). Bone morphogenetic protein 2 inhibits FSH responsiveness in hen granulosa cells. Reproduction (Cambridge, England) 140, 551-558.

Ji, H., Yang, H., Wang, J., Guo, J., Hu, Z., Zhang, H., Kong, F., Zhen, L., Guo, L. and Liu, J. (2014). Effect of adenovirus-mediated up-regulation of alpha-enolase gene products on follicle-stimulating hormone receptor mRNA and luteinizing hormone receptor mRNA of granular cells from goose F1 follicles. Research in veterinary science 96 , 526-532.

Jia, Y., Lin, J., Zeng, W. and Zhang, C. (2010). Effect of prostaglandin on luteinizing hormone-stimulated proliferation of theca externa cells from chicken prehierarchical follicles. Prostaglandins \& other lipid mediators 92, 77-84.

Johnson, A. L. and Woods, D. C. (2009). Dynamics of avian ovarian follicle development: cellular mechanisms of granulosa cell differentiation. General and comparative endocrinology 163, 12-17.

Kawabata, M., Imamura, T. and Miyazono, K. (1998). Signal transduction by bone morphogenetic proteins. Cytokine \& growth factor reviews 9, 49-61.

Knight, P. G. and Glister, C. (2003). Local roles of TGF-beta superfamily members in the control of ovarian follicle development. Animal reproduction science 78, 165-183.

Lam, K. M. (1997). Morphological evidence of apoptosis in chickens infected with infectious bursal disease virus. Journal of comparative pathology 116, 367-377.

Lee, Y. C., Cheng, C. J., Bilen, M. A., Lu, J. F., Satcher, R. L., Yu-Lee, L. Y., Gallick, G. E., Maity, S. N. and Lin, S. H. (2011). BMP4 promotes prostate tumor growth in bone through osteogenesis. Cancer research 71, 5194-5203.

Liu, H. Y. and Zhang, C. Q. (2008). Effects of daidzein on messenger ribonucleic Acid expression of gonadotropin receptors in chicken ovarian follicles. Poultry science 87, 541-545.

Livak, K. J. and Schmittgen, T. D. (2001). Analysis of relative gene expression data using real-time quantitative PCR and the 2(-Delta Delta C(T)) Method. Methods (San Diego, Calif.) 25, 402-408.

Makita, M. and Miyano, T. (2014). Steroid hormones promote bovine oocyte growth and connection with granulosa cells. Theriogenology 82, 605-612.

Massague, J. (2012). TGFbeta signalling in context. Nature reviews. Molecular cell biology 13, 616-630. 
Massague, J., Seoane, J. and Wotton, D. (2005). Smad transcription factors. Genes \& development 19, 2783-2810.

Massague, J. and Wotton, D. (2000). Transcriptional control by the TGF-beta/Smad signaling system. The EMBO journal 19, 1745-1754.

Mayeur, C., Kolodziej, S. A., Wang, A., Xu, X., Lee, A., Yu, P. B., Shen, J., Bloch, K. D. and Bloch, D. B. (2015). Oral administration of a bone morphogenetic protein type I receptor inhibitor prevents the development of anemia of inflammation. Haematologica 100, e68-71.

McGee, E. A. and Hsueh, A. J. (2000). Initial and cyclic recruitment of ovarian follicles. Endocrine reviews $21,200-214$.

Miyoshi, T., Otsuka, F., Inagaki, K., Otani, H., Takeda, M., Suzuki, J., Goto, J., Ogura, T. and Makino, H. (2007). Differential regulation of steroidogenesis by bone morphogenetic proteins in granulosa cells: involvement of extracellularly regulated kinase signaling and oocyte actions in follicle-stimulating hormone-induced estrogen production. Endocrinology 148, 337-345.

Nilsson, E. E. and Skinner, M. K. (2003). Bone morphogenetic protein-4 acts as an ovarian follicle survival factor and promotes primordial follicle development. Biology of reproduction 69 , 1265-1272.

Orisaka, M., Tajima, K., Tsang, B. K. and Kotsuji, F. (2009). Oocyte-granulosa-theca cell interactions during preantral follicular development. Journal of ovarian research 2, 9.

Pangas, S. A. and Matzuk, M. M. (2004). Genetic models for transforming growth factor beta superfamily signaling in ovarian follicle development. Molecular and cellular endocrinology 225, 83-91.

Qin, Q., Sun, A., Guo, R., Lei, M., Ying, S. and Shi, Z. (2013). The characteristics of oviposition and hormonal and gene regulation of ovarian follicle development in Magang geese. Reproductive biology and endocrinology : RB\&E 11, 65.

Regan, S. L., McFarlane, J. R., O'Shea, T., Andronicos, N., Arfuso, F., Dharmarajan, A. and Almahbobi, G. (2015). Flow cytometric analysis of FSHR, BMRR1B, LHR and apoptosis in granulosa cells and ovulation rate in merino sheep. Reproduction (Cambridge, England) 150, 151-163.

Schmierer, B. and Hill, C. S. (2007). TGFbeta-SMAD signal transduction: molecular specificity and functional flexibility. Nature reviews. Molecular cell biology 8, 970-982.

Su, D., Zhu, S., Han, X., Feng, Y., Huang, H., Ren, G., Pan, L., Zhang, Y., Lu, J. and Huang, B. (2009). BMP4-Smad signaling pathway mediates adriamycin-induced premature senescence in lung cancer cells. The Journal of biological chemistry 284, 12153-12164.

Tilly, J. L., Kowalski, K. I. and Johnson, A. L. (1991). Stage of ovarian follicular development associated with the initiation of steroidogenic competence in avian granulosa cells. Biology of reproduction 44, 305-314.

Tsukamoto, S., Mizuta, T., Fujimoto, M., Ohte, S., Osawa, K., Miyamoto, A., Yoneyama, K., Murata, E., Machiya, A., Jimi, E., et al. (2014). Smad9 is a new type of transcriptional regulator in bone morphogenetic protein signaling. Scientific reports 4, 7596.

Wang, W., Wang, L., Li, X. X., Chen, X., Zhang, H. Y., He, Y., Wang, J. J., Zhao, Y. Y., Zhang, B. L. and Xu, Y. X. (2010). Effect of interrupted endogenous BMP/Smad signaling on growth and steroidogenesis of porcine granulosa cells. Journal of Zhejiang University. Science. B 11, 719-727.

Wei, S., Chen, S., Gong, Z., Ouyang, X., An, L., Xie, K., Dong, J. and Wei, M. (2013). Alarelin active immunization influences expression levels of GnRHR, FSHR and LHR proteins in the ovary and 
enhances follicular development in ewes. Animal science journal = Nihon chikusan Gakkaiho 84, 466-475.

Xu, J., Li, J., Wang, H., Wang, G., Chen, J., Huang, P., Cheng, J., Gan, L., Wang, Z. and Cai, Y. (2015). A novel SMAD family protein, SMAD9 is involved in follicular initiation and changes egg yield of geese via synonymous mutations in exon1 and intron2. Molecular biology reports $\mathbf{4 2 ,}$ 289-302.

Ying, Y., Qi, X. and Zhao, G. Q. (2001). Induction of primordial germ cells from murine epiblasts by synergistic action of BMP4 and BMP8B signaling pathways. Proceedings of the National Academy of Sciences of the United States of America 98, 7858-7862.

Yu, P. B., Deng, D. Y., Lai, C. S., Hong, C. C., Cuny, G. D., Bouxsein, M. L., Hong, D. W., McManus, P. M., Katagiri, T., Sachidanandan, C., et al. (2008). BMP type I receptor inhibition reduces heterotopic [corrected] ossification. Nature medicine 14, 1363-1369.

Zhang, C. P., Yang, J. L., Zhang, J., Li, L., Huang, L., Ji, S. Y., Hu, Z. Y., Gao, F. and Liu, Y. X. (2011). Notch signaling is involved in ovarian follicle development by regulating granulosa cell proliferation. Endocrinology 152, 2437-2447. 


\section{Figures and figure legends}

\section{Figure 1}
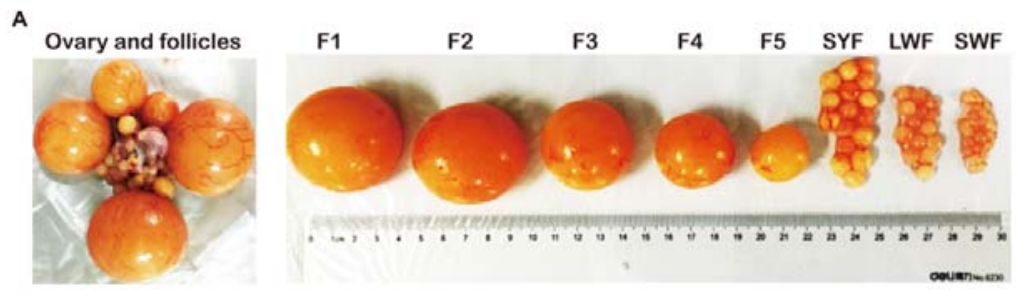

B
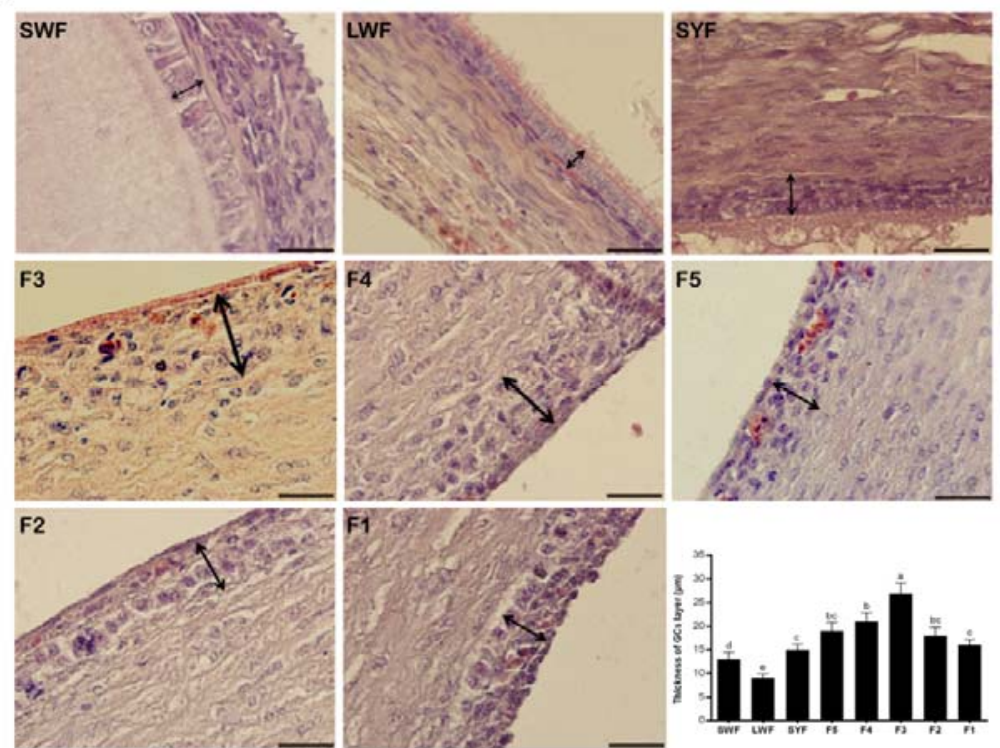

C
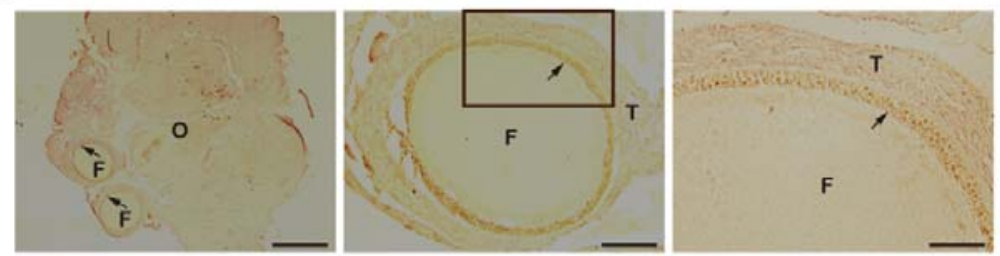

Fig. 1. Histological analysis of goose ovary and follicle in laying period and localization of Smad9 in goose ovary. (A) Ovary, hierarchical follicles and its size. (B) Variation and comparison of the thickness of GCs layer during follicular development; $n=3$. (C) Smad9 expression in the GCs layer of goose follicle. The letter $\mathrm{F}, \mathrm{O}$ and $\mathrm{T}$ represents the follicle, the ovary and the theca interna respectively. Arrows indicate Smad9 signaling in GCs layer. Bars with different lowercase letters indicate significant differences $(P<0.05)$. Scale bars: $20 \mu \mathrm{m}$ in B; $500 \mu \mathrm{m}$ in the left of $\mathrm{C} ; 100$ $\mu \mathrm{m}$ in the middle of $\mathrm{C} ; 50 \mu \mathrm{m}$ in the right of $\mathrm{C}$. 


\section{Figure 2}

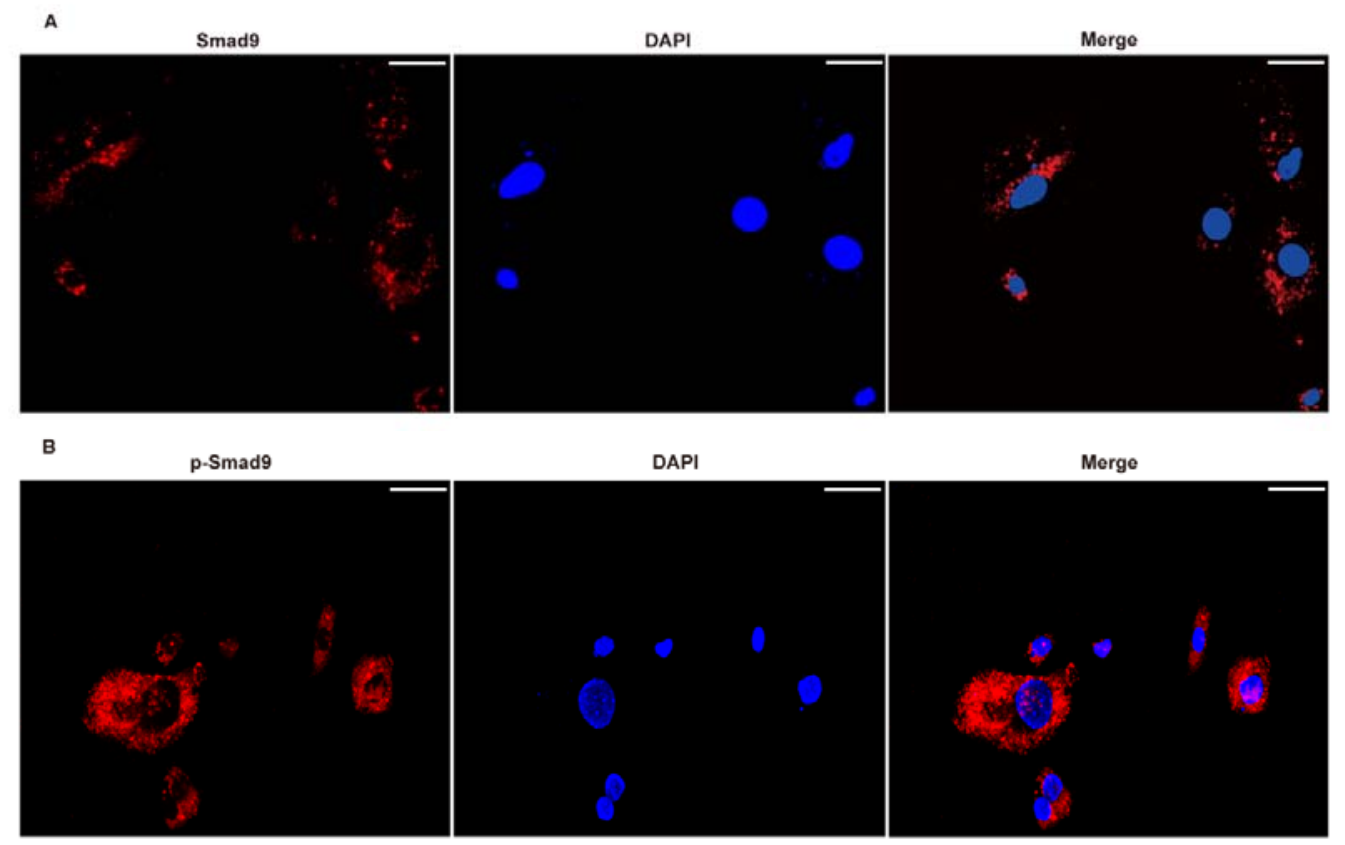

Fig. 2. Localization of Smad9 and p-Smad9 proteins in gGCs by immunofluorescence. Cells were fixed and stained with the nuclear dye 4,6-diamidino-2-phenylindole (DAPI; blue), red represents Smad9 or p-Smad9 signaling. (A) Smad9 signaling. (B) p-Smad9 signaling. Scale bars: $50 \mu \mathrm{m}$. 


\section{Figure 3}

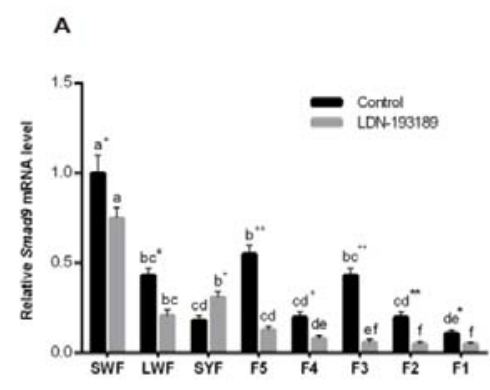

B

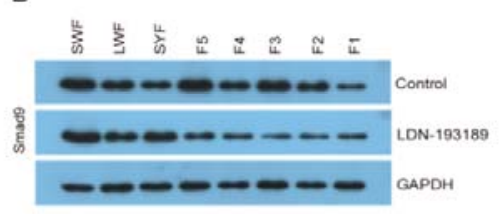

c
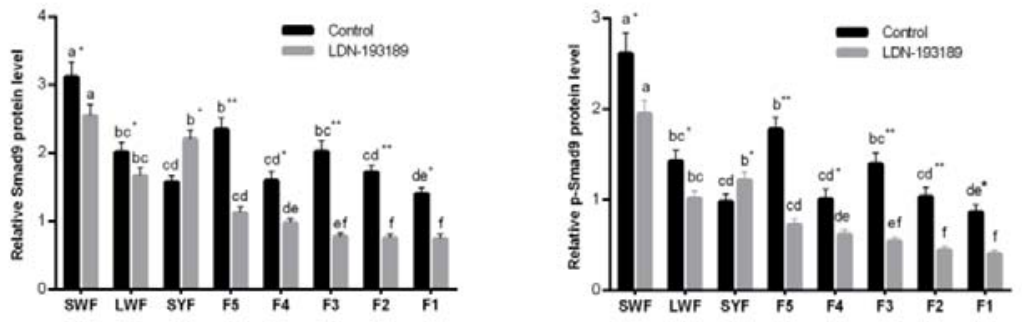

Fig. 3. Smad9 expression in LDN-193189 treatment and control groups in vivo.

(A) qRT-PCR analysis of Smad9 mRNA expression in LDN-193189 treatment and control groups. (B,C) Western blot analysis of Smad9 and p-Smad9 protein expression in LDN-193189 treatment and control groups. Bars with different lowercase letters are significantly different between the same group from different hierarchy follicles $(P<0.05)$ * and ** indicate significant differences between control group and LDN-193189 group in the same hierarchy follicles. * $P<0.05 ; * * P<0.01 ; n=3$. 


\section{Figure 4}
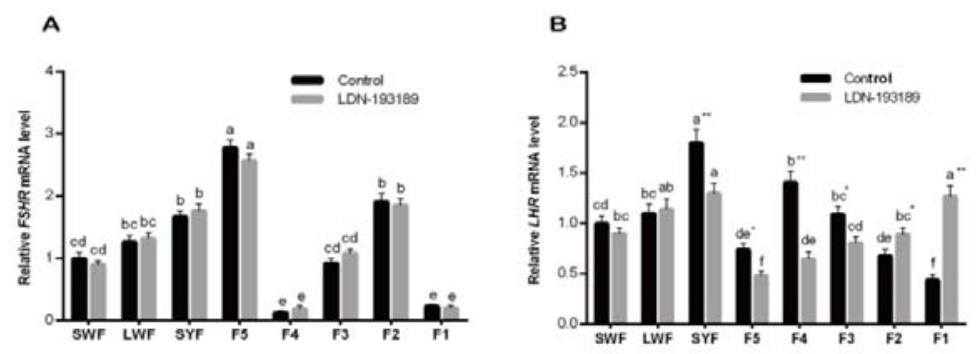

C

D
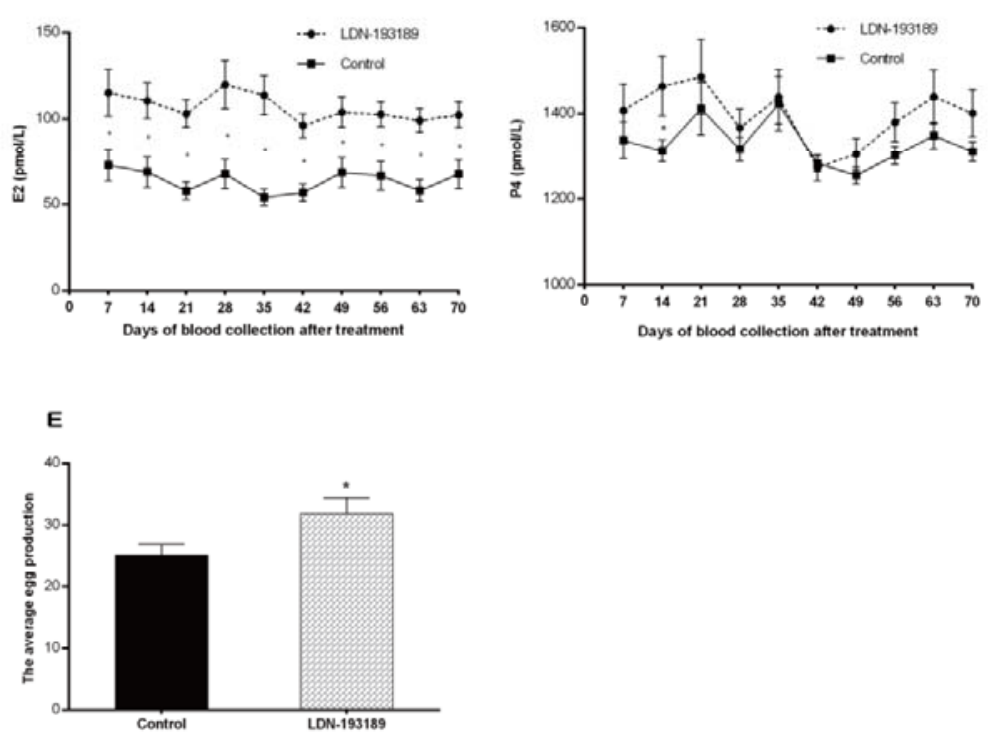

Fig. 4. FSHR and LHR expression, $E_{2}$ and $P_{4}$ secretion as well as the average egg production from LDN-193189 treatment and control groups in vivo. (A) qRT-PCR analysis of FSHR expression in LDN-193189 treatment and control groups. (B) qRT-PCR analysis of LHR expression in LDN-193189 treatment and control groups. (C,D) Determination of $\mathrm{E}_{2}$ and $\mathrm{P}_{4}$ expression level by ELISA in control and LDN-193189 groups. (E) The average egg production in control and LDN-193189 groups ( $n=10$ each). Bars with different lowercase letters are significantly different between the same group from different hierarchy follicles $(P<0.05) . *$ and $* *$ indicate significant differences between control group and LDN-193189 group in the same hierarchy follicles. $* P<0.05 ; * * P<0.01 ; n=3$. 


\section{Figure 5}
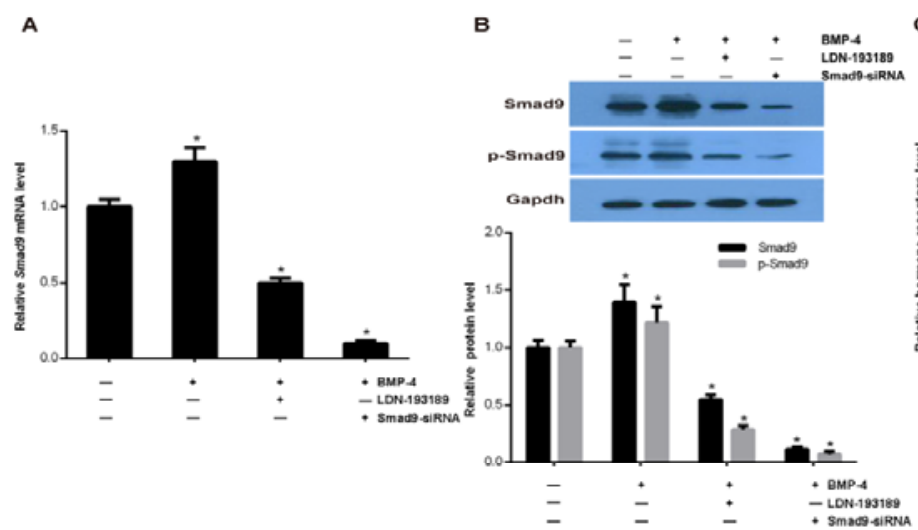

c

D

$E$
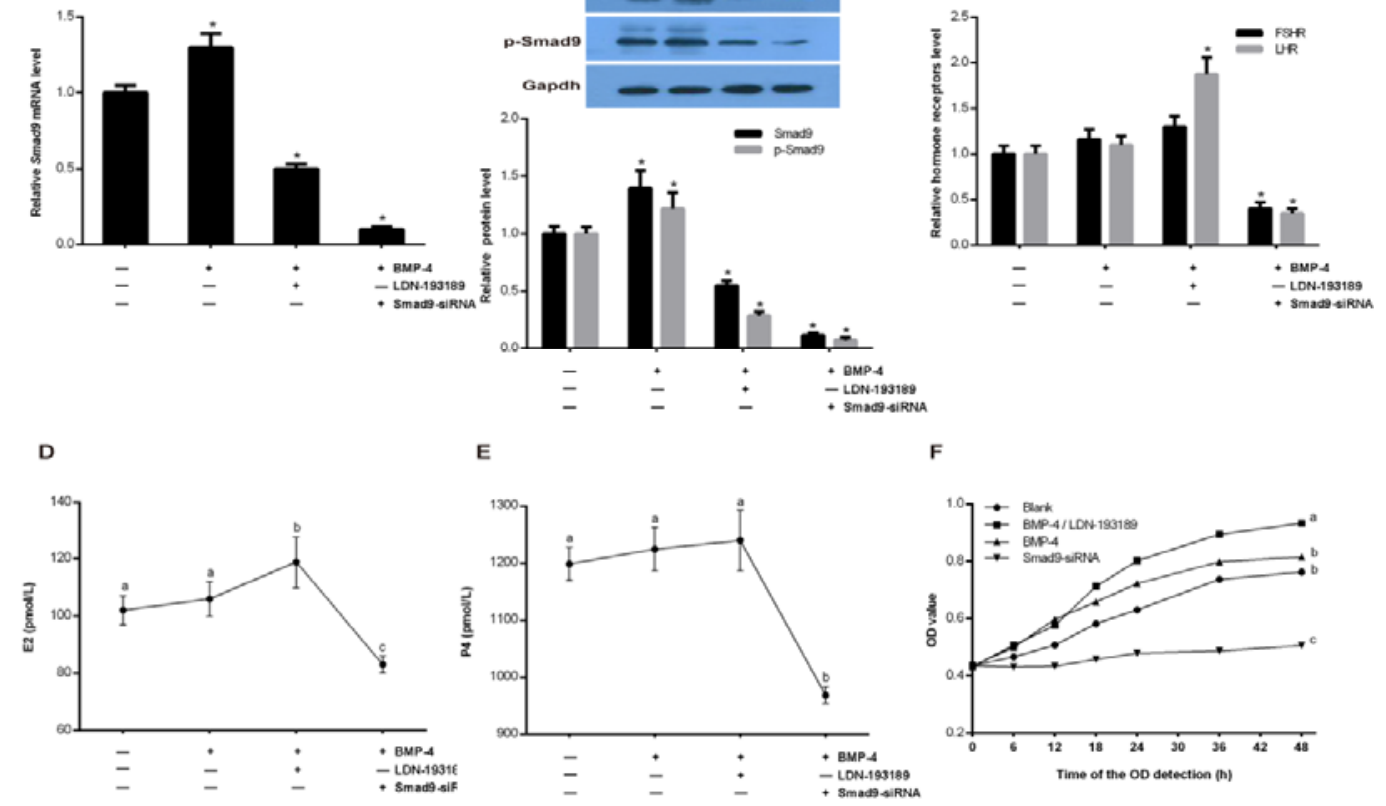

Fig. 5. FSHR and LHR expression, $E_{2}$ and $P_{4}$ secretion as well as cell proliferation are related to Smad9 expression which was treated by BMP-4, BMP-4 / LDN-193189 and Smad9-siRNA respectively for $48 \mathrm{~h}$ in cultured gGCs. (A) qRT-PCR analysis of Smad9 expression. (B) Western blot analysis of Smad9 and p-Smad9 proteins expression. (C) qRT-PCR analysis of FSHR and LHR expression. $(\mathrm{D}, \mathrm{E})$ ELISA measurement of $\mathrm{E}_{2}(\mathrm{D})$ and $\mathrm{P}_{4}(\mathrm{E})$ levels. $(\mathrm{F})$ Analyses of GCs growth using CCK-8 assay. Bars with different lowercase letters are significantly different between groups $(P<0.05) . *$ represents a significant difference compared to the blank group. $* P<0.05 ; n=3$. 


\section{Figure 6}

A
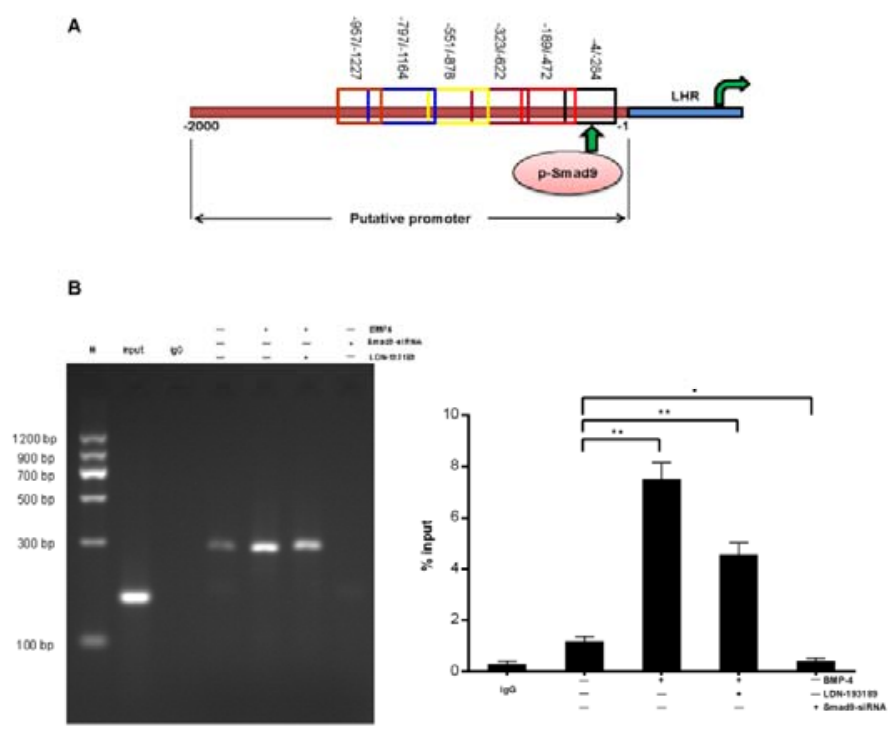

Fig. 6. Smad9 directly regulates LHR transcription in gGCs. (A) The cartoon shows the existence of Smad9 protein binding site at the upstream region of the LHR promoter. (B) The cell lysates from gGCs samples pull-down experiments showed different levels of enrichment in indicated groups. $* P<0.05 ; * * P<0.01 ; n=3$. 


\section{Figure 7}

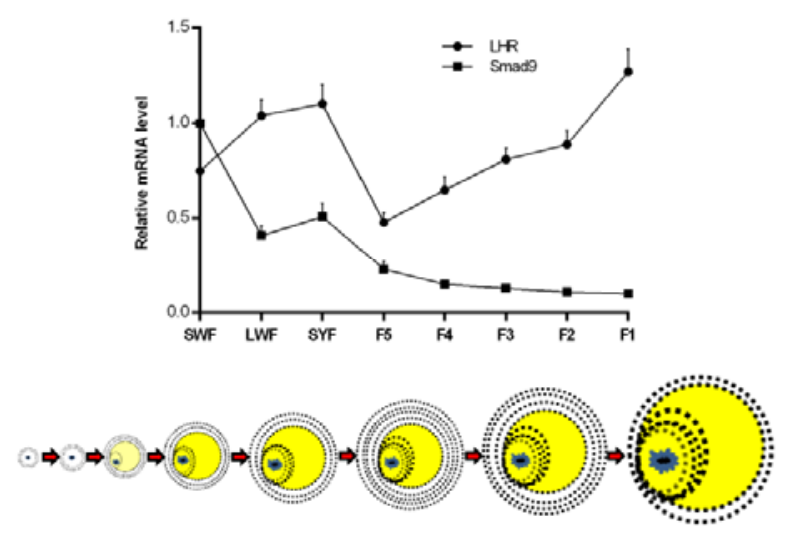

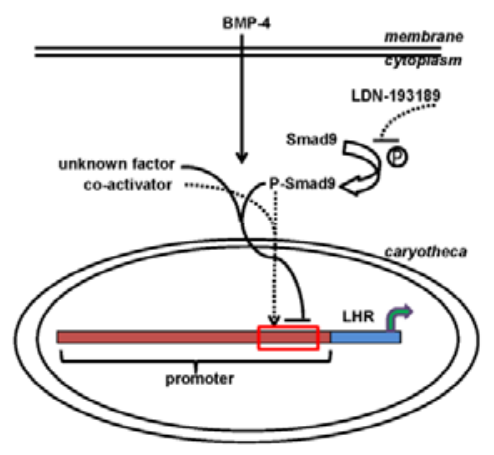

Fig. 7. The possible patterns and mechanisms of Smad9 regulating follicular development and ovulation in goose. (A) Smad9 expression and LHR transcription synergistic model with the development of follicles when Smad9 is suppressed. (B) One mechanism is conjectured by which Smad9, served as a sensor of balance, regulated LHR transcription. The solid line represents the control mode of the high expression of Smad9 and the dashed line represents the control mode when Smad9 is suppressed. 